\title{
Both Open and Closed NMDA Receptor Channels Desensitize
}

\author{
Fan Lin and Charles F. Stevens \\ Howard Hughes Medical Institute at The Salk Institute, La Jolla, California 92037
}

\begin{abstract}
Desensitization of NMDA receptors was studied at the single-channel level using outside-out patches taken from rat hippocampus CA neurons maintained short term in culture. The amount of desensitization that accumulated as a function of time during the application of NMDA $(2.5 \mu \mathrm{M})$ was measured by the response to the rapid application of NMDA at a high concentration $(100 \mu \mathrm{M})$. Records were sorted into two classes: those in which no openings were detected during the application of $2.5 \mu \mathrm{M}$ NMDA, and those in which some channel openings occurred. Analysis of these data reveals that some desensitization accumulated when no channels opened, but that more desensitization developed when channels opened. The observations indicate that channels can pass from the closed state to the desensitized state, but that desensitization occurs more rapidly from the open state.
\end{abstract}

[Key words: glutamate receptor, NMDA receptor, desensitization, single channel, kinetic analysis]

Glutamate receptors of the NMDA type are, like all receptors, driven into a desensitized state by the continuous presence of agonist. Because this receptor class exhibits slow responses to agonist presentation (Lester et al., 1990; Gibb and Colquhoun, 1991), entry into the desensitized state could be important in determining the overall time course of the receptor's response. Furthermore, understanding desensitization can be significant both for our appreciation of the receptor's role in brain function, and for elucidating the molecular mechanisms of its operation. Most authors have tentatively assumed that NMDA receptors make transitions from the closed to desensitized states (Benveniste et al., 1990; Clements and Westbrook, 1991; Clements et al., 1992; Lester and Jahr, 1992; Sather et al., 1992; Lester et al., 1993), but that open channels do not pass directly into the desensitized state. This assumption about which pathways can lead to desensitization has been rather arbitrary, however, as no direct evidence relating to permitted transitions is available. The conclusion of the work reported here is that both the open and closed states communicate with the desensitized state. In fact, we find that the open-to-desensitized transition is more rapid than that between closed and desensitized state.

The difficulty in studying pathways to desensitization is that closed states cannot be distinguished from desensitized states at the single-channel level: both states are characterized by the fact that no current flows. Two sorts of measurements can,

Received May 18, 1993; revised Sept. 17, 1993; accepted Sept. 29, 1993.

Correspondence should be addressed to Charles F. Stevens, The Salk Institute, 10010 North Torrey Pines Road, La Jolla, CA 92037.

Copyright (c) 1994 Society for Neuroscience $0270-6474 / 94 / 142153-08 \$ 05.00 / 0$ however, be made unambiguously to help define the state of receptors in a patch. First, the presence of a channel in the open state is detected by current flow, so the number of channels that are open as a function of time is - within the limits set by equipment resolution-directly measurable. Second, the probability that a channel is desensitized at a particular time after the start of agonist application can be estimated. This is accomplished by determining the average response of a population of receptors to a test pulse of agonist (a high agonist concentration rapidly applied) as compared to the response to the same test pulse when no desensitization has been produced; if, for example, the average response under one condition is half as large as that under a reference condition that produces no desensitization, the first condition desensitized half of the receptors. Note that this second method does not directly determine the number of receptors that are actually desensitized at a given time, but rather the fraction that are, on the average, in the desensitized state.

By making the two unambiguous measurements available, the number of channels in the open state and the average response to a test pulse of NMDA, the technique we use here can determine which pathways the receptor employs to reach the desensitized state in outside-out patches taken from cultured hippocampal pyramidal cells.

The idea behind our method is as follows: we apply a low concentration of NMDA (the prepulse) for various periods of time and then estimate the fraction of channels that have entered the desensitized state by the end of the prepulse from the size of the average response to a subsequent high-concentration pulse (the test pulse). The records are then sorted according to whether or not openings occurred during the prepulse. By applying the differential equations that describe the probabilistic behavior of NMDA receptor channels, we can use the additional information available-the probability that a channel is open during the application of desensitizing agonist-to identify the extent to which the closed-to-desensitized and the open-to-desensitized pathways are used. For example, any desensitization that accumulates during the prepulses for which no channels open must reflect operation of the closed-to-desensitized pathway. Alternatively, if more desensitization occurs during prepulses with channel openings than during those without, the open-to-desensitized transition must occur more rapidly than the closedto-desensitized transition.

\section{Materials and Methods}

Cell culture. Experiments were performed on primary cultures of hippocampal CA neurons prepared from 1-2-d-old neonatal Long-Evans rats, as described previously (Jahr and Stevens, 1987). Neurons were used between 15-72 hr after they were plated.

Single-channel recording. Single-channel recordings were made with the outside-out patch clamp configuration using a standard patch clamp amplifier (Axopatch-1A, Axon Instruments). Outside-out patch mem- 


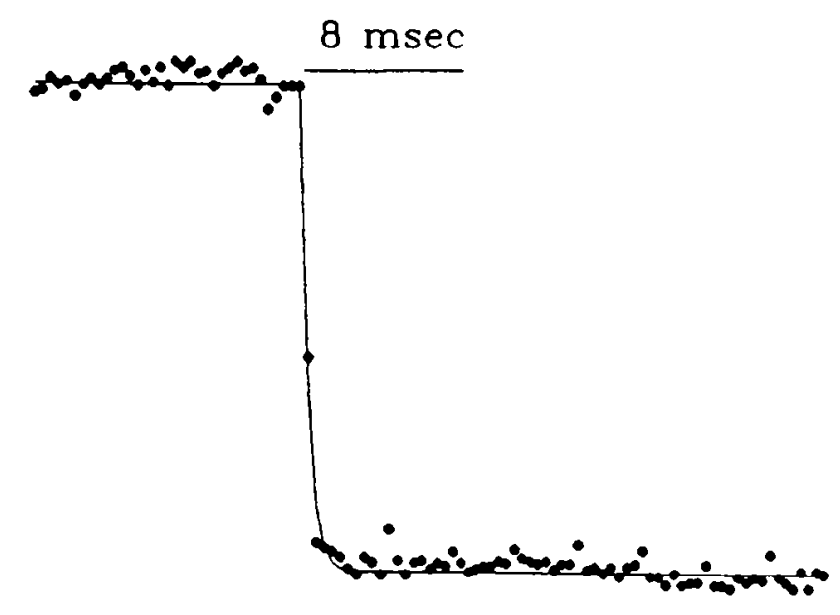

Figure 1. Fast solution change recorded using a concentration jump. The current change is recorded with an open patch pipette, clamped at $0 \mathrm{mV}$. The solution was switched from $20 \mathrm{~mm} \mathrm{NaCl}$ solution to 150 $\mathrm{mm} \mathrm{NaCl}$ solution and this caused $160 \mathrm{pA}$ to flow. The smooth curve is an exponential with a time constant of $0.4 \mathrm{msec}$.

branes were clamped at $-70 \mathrm{mV}$ and the currents recorded were lowpass filtered at $1 \mathrm{kHz}$. and digitally sampled at $2.5 \mathrm{kHz}$. The agonist application and data acquisition were directly controlled with a laboratory computer based on the Intel 80386 microprocessor. Usually the behavior of the patch was followed for 3-4 min after patch formation and any patches with spontancous channel activity under the continuous flow of the control bath solution were discarded. All the experiments were performed at room temperature $\left(20-21^{\circ} \mathrm{C}\right)$.

Solutions and drug delivery. Soft glass pipettes (Drummond), neither fire polished nor Sylgard coated, and with a resistance in range of 5-7 $\mathrm{M} \Omega$, were used to obtain the outside-out patches. The extracellular solution contained (in $\mathrm{mM}$ ) NaCl, $110 ; \mathrm{CaCl}_{2}, 2$; HEPES, 10; picrotoxin, 0.1 ; glycine, 0.002 ; tetrodotoxin (TTX), 0.001 ; and glucose, 10. TTX, Gly, and NMDA, which were kept frozen in separate stock solutions, were freshly added to make extracellular solution every $2 \mathrm{~d}$. The pipette solution was (in mM) $\mathrm{NaCl}, 5 ; \mathrm{CsCl}, 100$; tetraethylammonium, 20;1,2bis(2-aminophenoxy)ethane- $N, N, N^{\prime}, N^{\prime}$-tetra acetic acid (BAPTA), 10 HEPES, 10; $\mathrm{Mg}$-ATP, 2.5; and glucose, $10 . \mathrm{pH}$ was adjusted to 7.35 with $\mathrm{NaOH}$ in both solutions. All the chemicals were obtained from Aldrich, Alfa, or Sigma.

The drug delivery system consists of a four-barteled pipette, modified from those used by Carbone and Lux (1987) and Konnerth et al. (1987). The perfusion pipette was made of four plastic tubes, with diameters in the range of $250-300 \mu \mathrm{m}$. The four tubes were glued together and then glued into a larger enclosing capsule pipette of $5-6 \mathrm{~mm}$ in length. The glass capsule was made from patch pipette, but one-step pulled with a vertical pipette puller (Narishige, PP-83). The diameter of capsule tip opening was in the range of $200-250 \mu \mathrm{m}$ and the ends of the plastic tubes were about $500 \mu \mathrm{m}$ from the tip of the capsule. Three tubes were used to deliver agonist-containing solution, and the fourth was used only for control solution. Three-way solenoid valves (Neptune Research Inc.), situated between the four tubes and their solution reservoirs, were used to control the solution exchange. The valves were driven by a step pulse to open and close them abruptly. A $12 \mathrm{~V}$ driver circuit provided the step pulse and was controlled by the recording program via the digital output of an analog/digital output interface (TL-1, DMA Interface, Axon Instruments). Exactly one of the four valves controlling the perfusion pipette was always open so that the patch was continuously exposed in a flowing solution. The perfusion solutions were fed by gravity at about $0.5 \mathrm{ml} / \mathrm{min}$. In addition, the $0.5 \mathrm{ml}$ recording chamber was constantly perfused with the control bath solution at $2 \mathrm{ml} / \mathrm{min}$ to facilitate solution exchange. The time constant for solution exchange is less than 1 msec (Fig. 1).

Single-channel data analysis. The characteristics of our filter are such that the dwell time at half-maximum response is, in the noise-free case, equal to the open channel lifetime; for that reason, all open channel durations have been measured at the criterion level of $0.5 i_{u}$, where $i_{u}$ is the single-channel current (Colquhoun and Sigworth, 1983). In our analysis program, each current trace was previewed, and the threshold

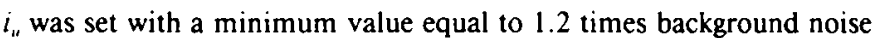
standard deviation; we reset this threshold as required during the analysis in order to take into account the events with different subconductance levels.

As we shall describe later, we need to estimate the probability that a channel is open during the agonist application. The probability that a channel is open at a specific time bin $(t)$ during the agonist presentation is calculated from the single-channel records according to the equation

$$
P_{s}\left(l_{t}\right)=\frac{1}{n N} \sum_{j=1}^{N} \sum_{k=1}^{M} \frac{l_{l, k}}{M_{i}},
$$

where $l_{y, k}$ is the number of channels open at the $k$ th sample point of $i$ th time bin in the $j$ th epoch. $M$, is number of sample points assigned to the $i$ th time bin with the shortest bins set equal to the sample interval $(0.4 \mathrm{msec})$ so that at least one sample point appears in them (see Fig. 5 caption). For the data presented in Figure $6, M_{1}=1$ for all time bins; that is, the bin width was always just one sample interval $(0.4 \mathrm{msec})$. For experiments of the sort illustrated in Figure $5 A$, however, the low agonist concentrations produced few openings: a brief, rapid transient of channel open probability occurs just after the onset of the prepulse, followed by a longer period of very low activity. In these experiments, the probability of a channel being open is calculated with bins that increase in length logarithmically during the prepulse. $N$ is number of epochs of agonist application; 37-132 individual epochs were used to construct the open probability distributions. $n$ is the estimated number of channels present in the patch. In an effort to determine the channel number present in the patch, we carried out nonstationary fluctuation analysis similar to that described by Sigworth (1980). This approach did not, however, yield an estimate for $n$ because plots of variance versus mean current passing through the NMDA receptor channel during the agonist application did not depart significantly from linearity. Therefore, we simply took our estimate for the number of channels present in the patch as the maximum number the channels open simultaneously during the entire recording period. This estimate provides a lower limit.

Unless otherwise indicated, all quantities are expressed as mean \pm SEM. The quality of all the data fits is based on the $\chi^{2}$ method (Press et al., 1989), with the programs written in Axobasic/Microsoft Basic.

\section{Results}

Agonist (NMDA) was applied to outside-out patches, taken from cultured hippocampal pyramidal cells, in two stages: first at low concentration (the prepulse) to produce desensitization, and then at high concentration (the test pulse) to determine what fraction of the channels occupied the desensitized state at the end of the prepulse. The prepulse terminated at the onset of the test pulse, and a 20-25 sec washing period was imposed after the test pulse ended before next prepulse was applied. This washing time was long enough to ensure the receptor recovery from the previous stimulus (unpublished observations). In order to minimize the influence of any "rundown" effects, control (prepulse concentration is zero) and prepulse recordings were alternated in a group of four or eight records. Repcated epochs of single-channel activity were recorded for the various combinations of prepulse concentrations and durations, and records were sorted according to whether NMDA receptor channel opening could or could not be detected during the prepulse. Our conclusions are based on studies of channels from more than 90 different patches with the prepulse agonist concentrations from $0,0.5,2.5$, to $5 \mu \mathrm{M}$ and prepulse durations that ranged from 0 to 2 sec. Test pulse concentration was 50 or $100 \mu \mathrm{M}$ and the test pulse duration was 1 or $2 \mathrm{sec}$. Different prepulse concentrations in the range between 0.5 and $5 \mu \mathrm{M}$, and test pulse concentrations of 50 and $100 \mu \mathrm{M}$ gave qualitatively the same results (Lin and Stevens, unpublished data); the detailed analysis presented here is based on patches with prepulse concentrations of 0 and $2.5 \mu \mathrm{M}$ and durations from 0 to $600 \mathrm{msec}$ in $100 \mathrm{msec}$ increments and with a test pulse concentration of $100 \mu \mathrm{M}$ and a duration of $1000 \mathrm{msec}$. 
A

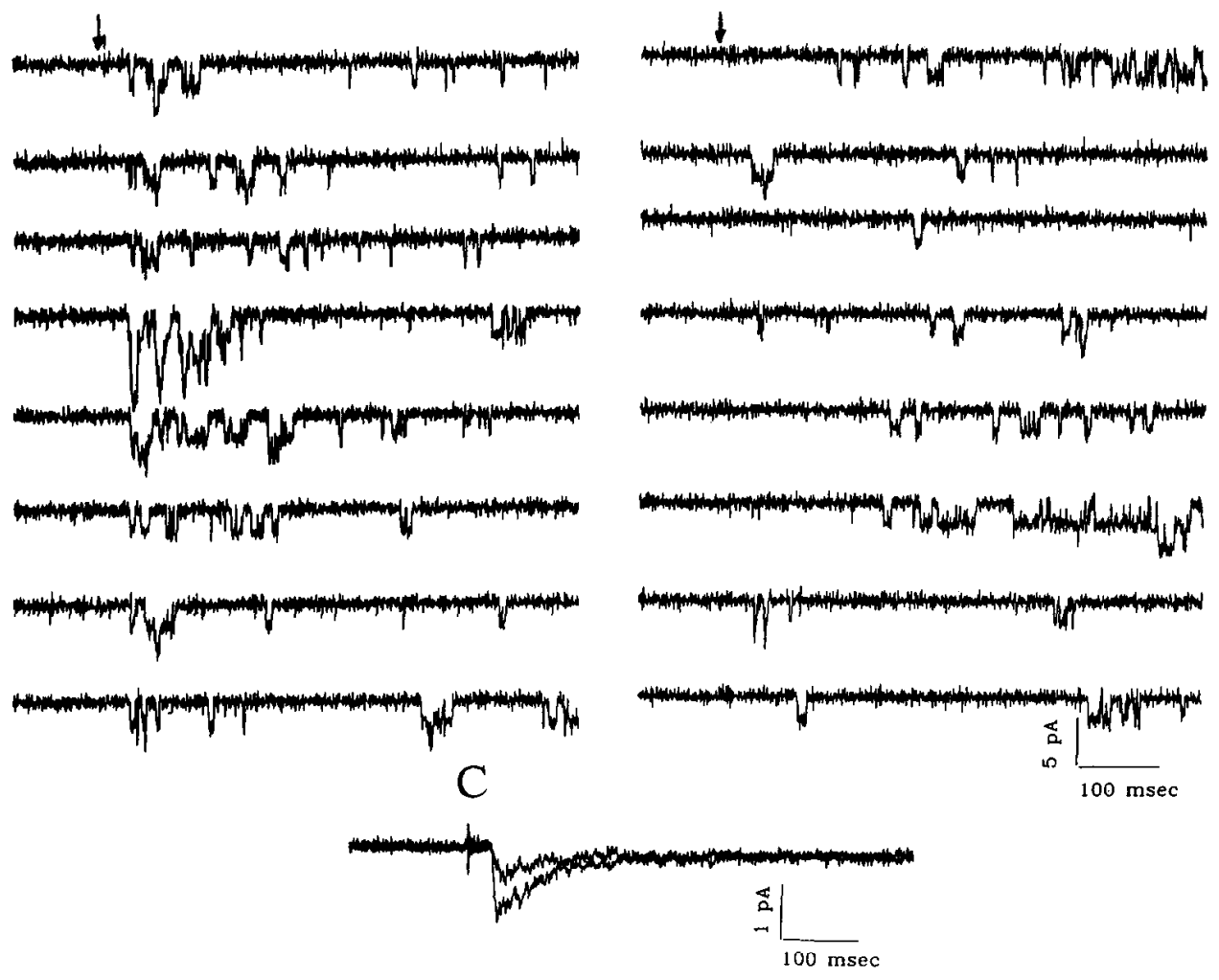

Figure 2. The NMDA receptor can reach its desensitized state without opening. $A$ shows the sample traces illustrating the response of a patch to the test pulse with prepulse NMDA concentration of zero (control). $B$ shows comparable sample traces for the response to a test pulse with $600 \mathrm{msec} 2.5$ $\mu \mathrm{M}$ NMDA prepulse; no openings were detected during the prepulse (only last $100 \mathrm{msec}$ shown). In $A$ and $B$ the test pulse was applied at the time indicated by the arrows. $C$ shows the ensemble average traces from the recording session that gave the sample traces shown in $A$ and $B$. There are 120 epochs for the control condition, and 105 with the prepulse present (but without prepulse openings). The control and prepulse recordings were always done alternatively to prevent the influence of "rundown." Probability of desensitization produced by the $600 \mathrm{msec}$ prepulse is 0.55 in this experiment.

\section{General observations}

Figure 2 illustrates sample records that illustrate the response to a test pulse after a $600 \mathrm{msec}$ prepulse with NMDA concentrations of 0 or $2.5 \mu \mathrm{M}$. The epochs of single-channel activity with a prepulse concentration of $0 \mu \mathrm{M}$ and $2.5 \mu \mathrm{M}$ are shown in Figure 2, $A$ and $B$. Any channel openings that occurred during the application of the agonist prepulse are called "prepulse openings"; if no openings were detected during the prepulse, we would designate the epoch as "no prepulse openings." Sample epochs with no prepulse openings are shown in Figure $2 B$. Because "rundown" of NMDA receptor currents can occur under the conditions of our experiments, we alternated between the various prepulse conditions. All of the records with the same prepulse concentration, duration, and category (prepulse openings, or no openings) were averaged together; ensemble averages are illustrated in Figure $2 C$.

To determine if NMDA receptors can enter the desensitized state from both its closed and open states, we compared responses (samples illustrated) to test pulses of NMDA after application of prepulses with either 0 (control, Fig. $3 A$ ) or $2.5 \mu \mathrm{M}$ NMDA (Fig. $3 B, C$ ). Openings never occurred during the control $(0 \mu \mathrm{M})$ NMDA prepulse, but some prepulse applications with $2.5 \mu \mathrm{M}$ NMDA resulted in prepulse openings and some did not. We sorted records into those in which no prepulse openings were detected (Fig. $3 B$ ) and those with at least one prepulse opening (Fig. $3 C$ ). Ensemble averages from these three classes of records are exhibited in Figure $3 D$ with averages for the control (a), no prepulse opening (b), and at least one prepulse opening (c) classes. Clearly some desensitization developed with no prepulse openings occurred (compare Fig. $3 D$, a and b), so the closed channels are desensitized by $2.5 \mu \mathrm{M}$ NMDA. But even more desensitization accumulated during the prepulse period when channels did open (compare Fig. 3D, b and c), so open channels pass more readily into the desensitized state than do closed channels.

We note here two problems with this interpretation that will be revisited later. First, some undetected, very brief openings undoubtedly occurred during the prepulses in the no-openings category. We need to deal with the question of whether the desensitization we have attributed to the closed-to-desensitized transition actually used the open-to-desensitized pathway through the unobserved open states. Second, we have attributed the extra desensitization seen with prepulse openings to the open-to-dcsensitized transition, whereas the occurrence of openings might logically have simply altered the closed-to-desensitized rate. That is, a closed channel that previously has been open might remember its history and desensitize more rapidly than a closed channel that has not opened recently.

\section{No prepulse openings}

For a quantitative evaluation of our data, we consider the simplified situation in which the NMDA receptor can be considered to exist in only three states (in addition to the resting state without agonist bound to it): $\mathrm{C}=$ closed, $\mathrm{O}=$ open, and $\mathrm{D}=$ desensitized. A more complete treatment doubtlessly would require additional states, but these three compound states seem adequate for our present purposes. The state diagram for the receptor at this level of analysis, then, is

$$
\mathrm{C} \leftrightarrow{ }_{\mathrm{D}} \mathrm{O}
$$



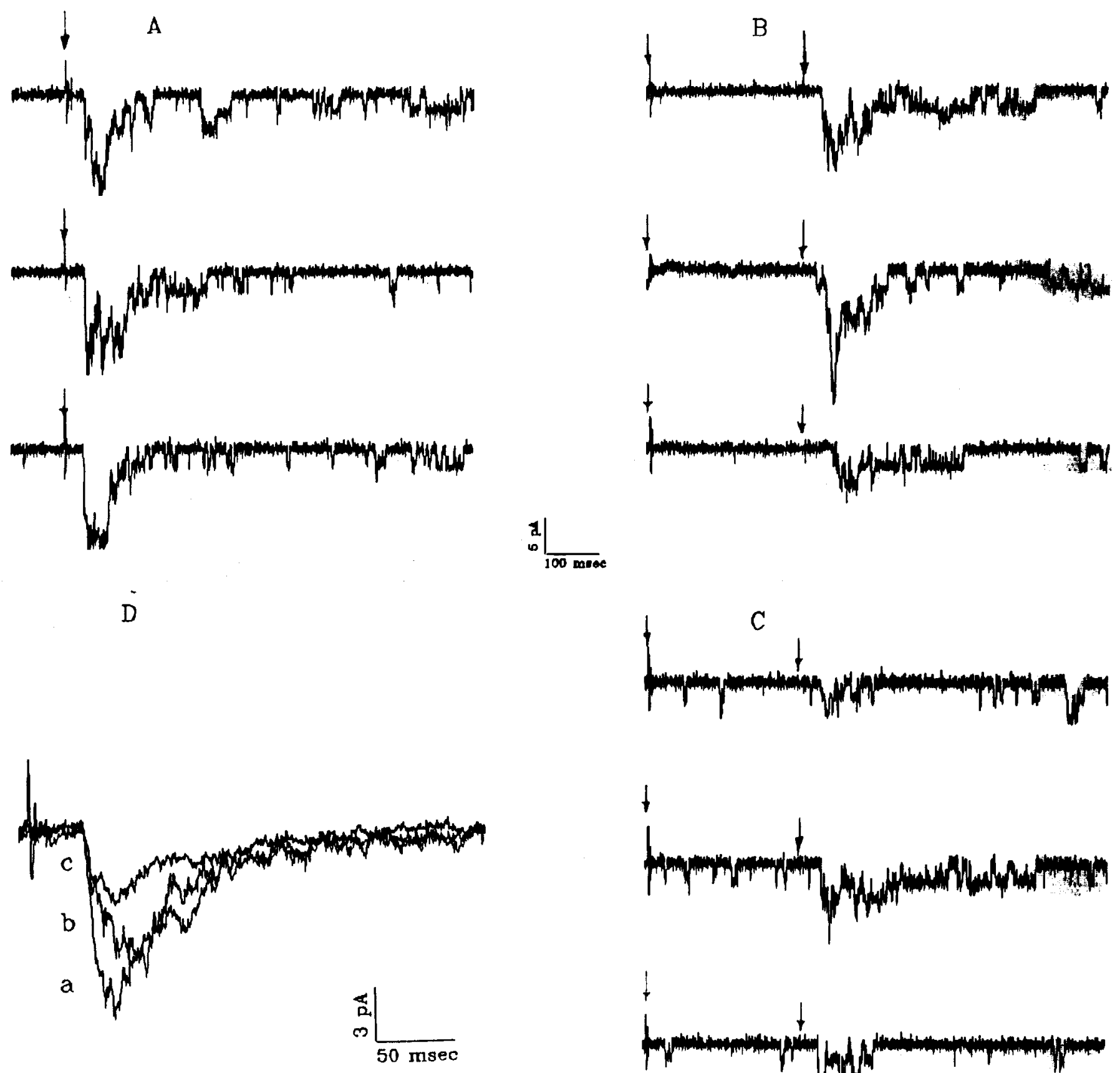

Figure 3. The NMDA receptor desensitized state can be reached from both closed and open states: sample records of current as a function of time for the control condition $(A)$ and after a $300 \mathrm{msec}$ prepulse with $2.5 \mu \mathrm{M}$ NMDA $(B$ and $C)$. Sample records in $B$ had no openings detected during the prepulse and those in $C$ had at least one opening during this period. Arrows indicate the onset of both prepulse and test pulse. In $D$, ensemble averages of the response to the test pulse are shown. $a$, Presents the average of 13 control traces. For $b$ (average of 12 traces) and $c$ ( 16 traces), $2.5 \mu \mathrm{M}$ NMDA was present for $300 \mathrm{msec}$ before the test pulse presentation, but no prepulse openings were detected for $b$, but at least one prepulse opening was present for each of the traces that contributed to $c$. Test pulse concentration was $100 \mu \mathrm{M}$.

where the $\mathrm{C} \rightarrow \mathrm{D}$ rate constant is $\alpha$, the $\mathrm{D} \rightarrow \mathrm{C}$ rate constant is $\beta$, the $\mathrm{O} \rightarrow \mathrm{D}$ rate constant is $a$, and the $\mathrm{D} \rightarrow \mathrm{O}$ rate constant is $b$. We shall not be directly concerned with the kinetics of the $\mathrm{C} \leftrightarrow \mathrm{O}$ transition.

We first consider the situation in which no prepulse openings occur. Our goal is to derive an equation for the probability $P_{D}$ that a channel is in the desensitized state (this probability is estimated by the response to the test pulse). The differential equation that governs the probability $P_{D}$, occupancy of the $\mathrm{D}$ state for the state diagram (2), is

$$
\frac{d P_{D}}{d t}=-(b+\beta) P_{D}(t)+a P_{o}(t)+\alpha P_{c}(t)
$$

where $P_{o}$ is the probability of the open state occupancy and $P_{c}$ 
is the rabability of closed state occupancy. For those epochs in when no prepulse openings occurred, $P_{o}$ is zero throughout the prepare, so $P_{c}+P_{D}=1$. Use this last relation (together with $\boldsymbol{E}_{0}=\mathbf{0}$ )- to simplify the differential Equation 3 to give

$$
\frac{d P_{D}}{d t}=-(\alpha+b+\beta) P_{D}(t)+\alpha .
$$

Thastist equation predicts the fraction of receptors in the desempitized state as a function of time after the start of the prefolstis $p_{D}$ can be estimated from the average response to a test pialse: Assume that initially no receptors are desensitized. Therion to the equation is then

$$
P_{D}(t)=P_{\mathcal{X}}(1-\exp (-(\alpha+b+\beta) t)),
$$

where $P_{f}=\alpha /(\alpha+b+\beta)$ is the probability of a receptor being in thensitized state after a very long prepulse. Call $\tau=1 /(\alpha$ ++ Since both $P_{f}$ and $\tau$ can be measured from the size of the response to the test pulse after various prepulse durations (seebelow), we can (1) test the adequacy of this formulation for deseribing the accumulation of desensitization during the prepulan (2) estimate the $\mathrm{C} \rightarrow \mathrm{D}$ rate constant $(\alpha)$, and the sum of rate constants $(b+\beta)$ for leaving the desensitized state.

The probability of desensitization $\left(P_{D}\right)$, that is, the probability that a receptor would be found in the desensitized state after a particular prepulse, is calculated from the average transmembranerarge movement during the first $100 \mathrm{msec}$ of the test

$$
\begin{aligned}
\text { pulanse } & \left(Q_{100}=\int_{0}^{100} I(t) d t\right) \text { as } \\
P_{D} & =1-\frac{Q_{100}(\text { prepulse })}{Q_{100}(\text { control })} .
\end{aligned}
$$

Foreach $P_{D}$ data point, the $Q_{100}$ values for both prepulse and control were obtained from the same neuron. The probability of desensitization that occurs by the end of the prepulse with no openings is shown as a function of prepulse duration in Figure 4. which presents data points obtained with Equation 5. The patiotions of Equation 4 are satisfactory, and the $C \rightarrow D$ rate constant $(\alpha)$ is estimated to be $2.1 \mathrm{sec}^{-1}$. The total rate constant forteayng the desensitized state $(b+\beta)$ is $1.1 \mathrm{sec}^{-1}$.

\section{Prepulse openings}

Whopenings occur during the agonist prepulse, the receptor has the opportunity to desensitize from either the closed or the open states. The differential equation that describes this process again is Equation 3. The conservation of probability requires that $P_{B}+P_{o}+P_{c}=1$, so elimination of $P_{c}$ from Equation 3 for this situation gives

$$
\frac{d P_{D}}{d t}=-(a+b+\alpha+\beta) P_{D}(t)+\alpha-a P_{o}(t)
$$

Because openings occur during the prepulse, the term for the probability of the open state $P_{\nu}$ naturally still appears. We can, however, estimate the probability of the open state occupancy as function of prepulse duration, $P_{o}(t)$, directly from the experimental records by simply reconstructing the open event distribution (Eq. 1). With the estimate for $P_{o}(t)$ available, we can solve the Equation 6 to give

$$
P_{D}(t)=\int_{0}^{t} e^{\cdots\left(\ell^{\prime}\right) / \tau}\left(\alpha-a P_{O}\left(l^{\prime}\right)\right) d l^{\prime}
$$

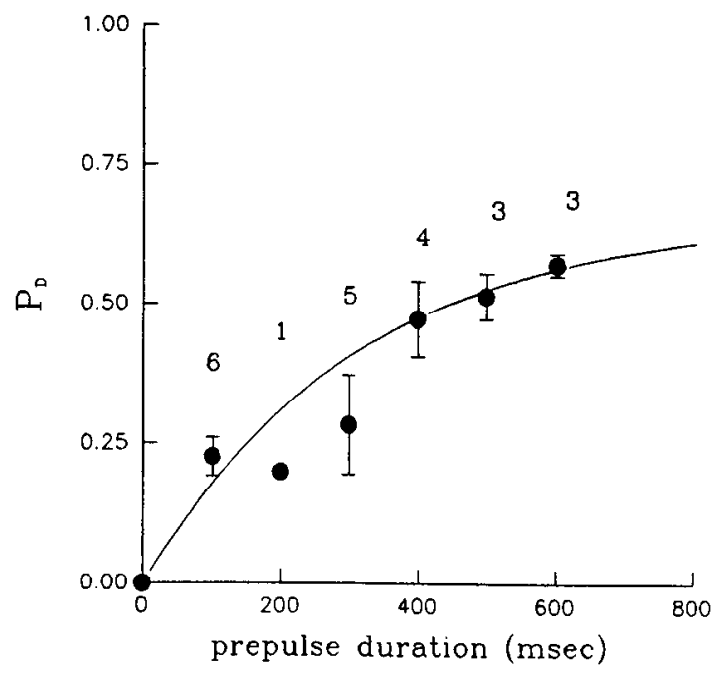

Figure 4. Probability of the desensitized state as a function of time for closed channels. The desensitization accumulated $\left(P_{D}\right)$ during a specific prepulse was obtained according to Equation 5 . The prepulse durations range from 0 to $600 \mathrm{msec}$. Traces were selected to have no prepulse openings. Each data point was obtained from one to six different patches for the same prepulse duration; the numbers above the trace specify the number of patches that contributed to each data point shown. The continuous line is the best-fitting curve from Equation 4. The rate constant for $\mathrm{C} \rightarrow \mathrm{D}(\alpha)$ is estimated to be $2.1 \mathrm{sec}^{-1}$. The rate constant for leaving the $\mathbf{D}$ state $(\beta+b)$ is $1.1 \mathrm{sec}^{-1}$.

where the time constant is now defined as $\tau=1 /(a+b+\alpha+$ $\beta)$.

An upper limit for the open probability $P_{n}$, shown as data points in Figure $5 A$, was determined with Equation 1, from an ensemble average of 38 epochs, each with at least one opening. NMDA concentration was $2.5 \mu \mathrm{M}$ with a 2 sec duration. An estimate $n$ for (the lower limit of) the number of channels present in the patch was taken as the maximum number of channels simultaneously open observed during $100 \mu \mathrm{M}$ NMDA application in the same neuron. The open probability function was determined empirically and is displayed in Figure $5 A$ as a superimposed smooth curve that describes the data.

The equation for $P_{D}(t)$ is easily solved for the empirical driving function $P_{o}(t)$ to give Equation 7 . The only unknown quantity $a$ was varied (note that $\alpha$ and $b+\beta$ have already been estimated in the previous analysis) until the best fit between data points and $P_{D}(t)$ function was found. Figure $5 C$ shows the experimental data (data points) and the theoretical fit (curve a) determined in this way. The rate constant $a$ is estimated to be $50.2 \mathrm{sec}^{-1}$ (compare to the value of $\alpha$ as $2.1 \mathrm{sec}^{-1}$ for the desensitization rate from the closed state, curve c). Thus, the open-desensitized transition occurs with a rate greatly exceeding that for the closeddesensitized transition.

\section{Effects of undetected openings}

Some undetected openings undoubtedly occurred during the epochs classified as no-prepulse openings. We now evaluate the effects of such undetected openings on our estimates of rate constants.

In order to estimate the fraction of undetected openings, we have assumed that the open times are exponentially distributed in $0-25 \mathrm{msec}$ range and have fitted the exponential distributions to the portion of the open time histograms not appreciably altered by limitations in recording bandwidth, as shown in Fig- 

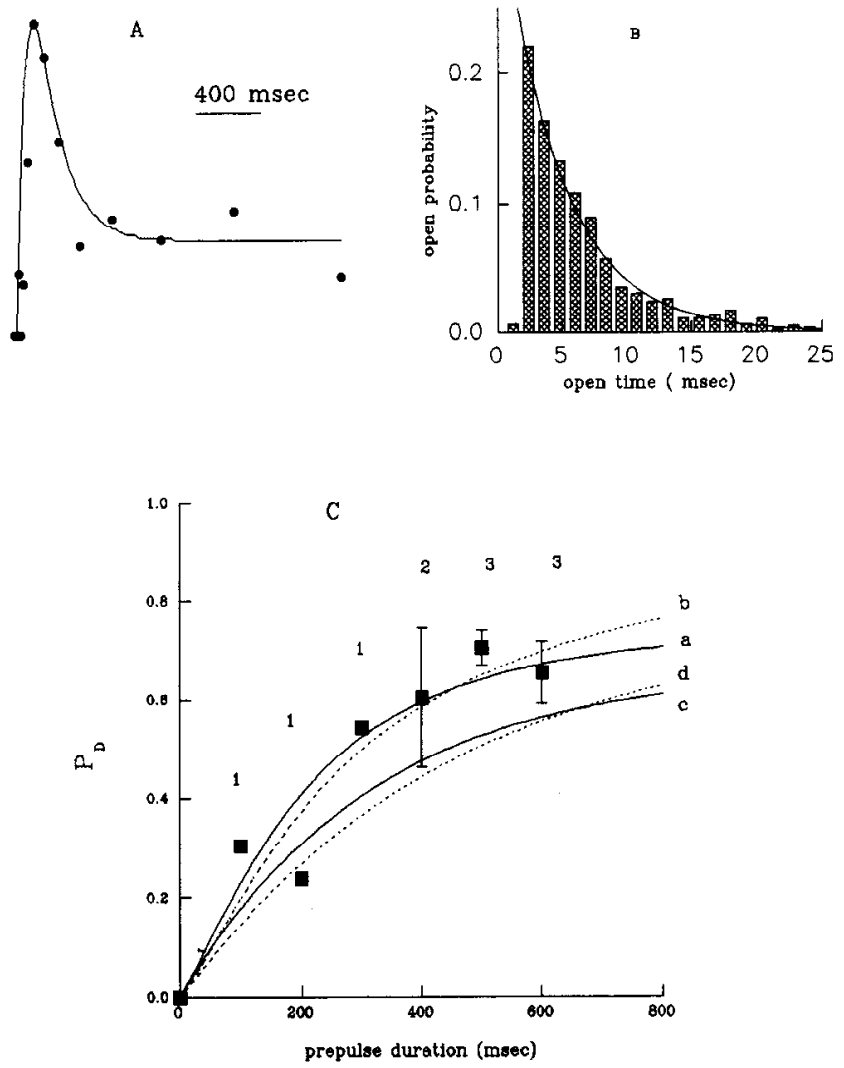

Figure 5. Probability of the desensitized state as a function of time with channel opening. $A$, To solve the problem shown in Equation 6 , the open probability as function of time during the $2.5 \mu \mathrm{M}$ NMDA application was empirically determined as $P_{o}(t)=0.0407(1-\exp (-t /$ $55))-0.0357(1-\exp (-t / 147.1))$, shown as the smooth curve. The open probability distribution is constructed from responses to a $2 \sec 2.5 \mu \mathrm{M}$ NMDA application according to Equation 1, shown as data points. Thirty-eight epochs were used to construct this distribution. Logarithmic binding has been used such that the 2000 -msec-long pulse time is divided into 22 bins. $B$, The fraction of missed events can be estimated from the difference between the observed open time histogram (bars) and the exponential distribution (smooth curve). For the patch illustrated, the fraction of missed events was estimated to be 0.1 . $C$, The data points specify the amount of desensitization accumulated due to the transition among $\mathrm{C}, \mathrm{D}, \mathrm{O}$ states during prepulses with different durations. The number of patches that contributed to each point is indicated by the numbers above the trace in the figure. Epochs with at least one prepulse opening were used to construct the ensemble average. The prepulse agonist concentration is $2.5 \mu \mathrm{M}$ and test pulse concentration is $100 \mu \mathrm{M}$. The solid curve a given by Equation 7 as described in text. The rate constant from $\mathrm{O} \rightarrow \mathrm{D}(a)$ is estimated to be $50.2 \mathrm{sec}^{-1}$. Solid curve $c$ is the one that appears in Figure 4 to describe the probability of desensitization by the prepulse when no openings are detected. The data were refitted after correcting the effect of missing events and shown as a dashed curve $b$ for prepulse openings, and as dashed curve $d$ for no prepulse openings (see Results).

ure $5 B$. The integral of the difference between the observed open time histogram and the theoretical exponential distribution then provides an estimate for $\theta$, the fraction of openings that were missed. For 20 patches the average value for $\theta$ was $0.174 \pm$ 0.013 , with the range from 0.03 to 0.25 .

We are therefore able to estimate the actual open probability $\left(P_{\text {od }}\right)$ during prepulse period when openings were detected, as

$$
P_{\text {od }}=\frac{P_{o}}{1-\theta}
$$

where $P_{o}$ is the apparent probability of a channel being open

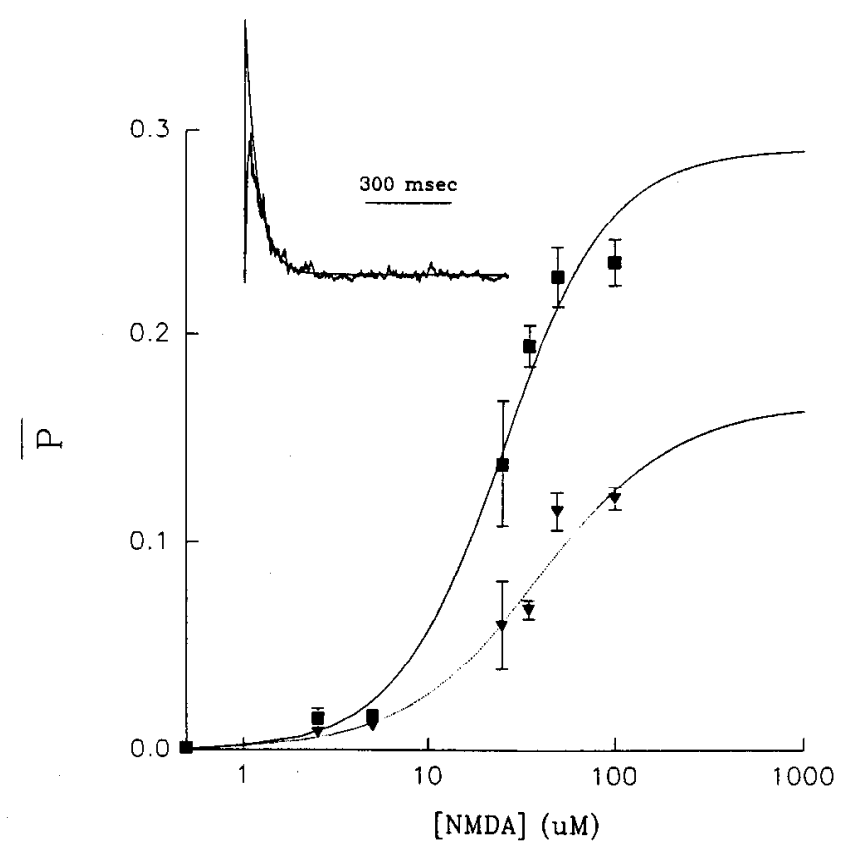

Figure 6. Peak open probability $(\bar{P})$ as function of NMDA concentration. Both experimentally observed peak open probability (triangles) and extrapolated values (squares) are shown. The data points were obtained from 2 to 10 neurons for each concentration. Observed $\bar{P}$ was directly obtained from open probability distribution constructed with Equation 1. Extrapolated peak open probability $\bar{P}$ was obtained by extrapolating the declining phase of open probability distribution to the onset of response, as shown in inset. The smooth lines are the fitted curves from Equation 8. The maximum open probability at saturated NMDA concentration was estimated to be 0.16 for observed peak probability and 0.29 for the extrapolated one. $K$ and $M$ were estimated as (mean $\pm \mathrm{SD}$ ) $31.75 \pm 3.05 \mu \mathrm{M}$ and $2.44 \pm 0.16$ from extrapolated peak $\bar{P} ; 45.88 \pm 7.50 \mu \mathrm{M}$ and $4.99 \pm 0.45$ from observed peak $\bar{P}$. Hill constant $(\mathrm{h})$ is 1.5 for the extrapolated open probability and 1.2 for the observed one.

during the prepulse when openings were detected. Call $P_{\text {on }}$ the actual probability of a channel being open during the prepulse period when no openings were detected. It is estimated as $P_{\text {on }}$ $=\theta P_{\text {od }}$.

We have used Equation 7, with $P_{\text {on }}$ or $P_{\text {od }}$ replacing the function $P_{o}$ that appeared before, to find the best fit to the data points in Figures 4 and $5 C$. The final fitted curves are shown as the dashed lines in Figure $5 \mathrm{C}$. The starting estimates for rate constants were the ones determined in the two preceding sections, and the parameter space was searched until two sets of data in Figure 4 and $5 C$ were best fitted by a single set of rate constants. The values found are $\alpha=1.5 \mathrm{sec}^{-1}$ for the closed to desensitized transition (curve d; compare to $2.1 \mathrm{sec}^{-1}$ estimated earlier, shown in curve c and Fig. 4) and $a=47.3 \mathrm{sec}^{-1}$ for the open to desensitized transition (curve b; compare to value of $50.2 \mathrm{sec}^{-1}$ estimated earlier, curvc a). The new estimate for the rate of leaving the desensitized state is $b+\beta=0.5 \mathrm{sec}^{-1}$ as compared to the earlier estimate of $1.1 \mathrm{sec}^{-1}$. Thus, the corrections for missed events do modify the rate constants slightly but, as would be expected, do not have a pronounced effect.

\section{Maximum open probability}

In the course of these studies we have accumulated data on the response of patches to NMDA concentrations from 0.5 to 100 $\mu \mathrm{M}$, and we can use these data to estimate the maximum open probability of a channel for high NMDA concentrations. We 
have estimates for the probability that a channel is open as a function of time after the onset of an agonist application, and so we know the peak probability that the channel is open. The problem is that desensitization occurs quite rapidly at high agonist concentrations, and this causes the peak of the curve relating open probability to time to be attenuated. If we assume that desensitization approximately follows a single exponential time course and that the opening kinetics are rapid on the time scale set by this desensitization time constant, we can extrapolate the declining current to zero time to obtain the probability of a channel being open if no desensitization had occurred. An example of this extrapolation is illustrated in the insert of Figure 6. The observed peak probability (triangles) and that estimated by extrapolation (squares) are presented as the data points in Figure 6 . Here, both curves have been fitted by the equation

$$
\bar{P}=\frac{1}{1+M\left(1+\frac{K}{C^{h}}\right)}
$$

where $\bar{P}$ is the peak open probability, $C$ the NMDA concentration, and $\mathrm{h}$ the Hill coefficient. $K$ reflects the ratio of rate constants between closed and resting states and $M$ the ratio of rate constants between opened and closed states; $M$ determines the maximum open probability. The maximum open probability $(\bar{P})$ at saturated agonist concentrations was estimated to be 0.16 for observed peak open probability (this gives the lower limit on the true value) and 0.29 for extrapolated one (our estimate for the true maximum open probability; see Fig. 6).

\section{Discussion}

Use of single-channel recording data to determine a channel's state diagram and associated rate constants is always difficult (McManus et al., 1989). The approach we have adopted here circumvents much of this difficulty for our particular situation by exploiting experimenter's ability to sort records according to some particular characteristic (here, no prepulse openings) and to assay the total occupancy of a state (from the test pulse).

Our main conclusion is that desensitization can occur from both the open and closed states, but that the rate of desensitization from the open state is much higher than that from the closed state. This conclusion applies only to the calcium-, glycine-insensitive form of desensitization (Sather et al., 1990; Shirasaki et al., 1990; Lester et al., 1993); the calcium-sensitive form (Mayer and Westbrook, 1985; Legendre et al., 1993) was presumably minimized in our experimental condition (we used isolated patches and $10 \mathrm{~mm}$ BAPTA in the pipette solution). Furthermore, the glycine-sensitive form (Mayer et al., 1987) also appears to be absent because we used saturating concentrations of glycine in the external solution, and because the glycine-sensitive form of desensitization is generally lost after the formation of isolated patch (Sather et al., 1990; Lester et al., 1993). We cannot, of course, be sure that we were investigating only a single type of desensitization. Perhaps two distinct desensitization mechanisms are present in our patches, one that operates on closed channels and another on open channels. The observation that we, like others (Sather et al., 1992), detect only a single time constant for the development of desensitization argues in favor of a single mechanism, but does not rule out the alternative, more complicated possibility of multiple forms.

Our observation of desensitization from the closed state confirms and extends the observations of Chizhmakov et al. (1992),
Zilberter et al. (1991), and Lester et al. (1993). These authors found that aspartate, NMDA, and glutamate would produce desensitization even when channel opening was prevented because glycine was absent, but that glycine alone produced no desensitization. In these experiments using whole-cell recording, then, channels entered the desensitized state without detectable openings (although the actual number of openings that occurred during the prepulse are difficult to estimate from the data presented in the reports). Our observations directly demonstrate that desensitization can occur without channel openings when NMDA and glycine are both present. The fact that desensitization is more rapid for open channels also might explain the finding of Chizhmakov et al. (1992) that glycine, together with aspartate, produces a greater degree of desensitization than aspartate alone; when both amino acids are present, channels have the opportunity to desensitize from the open state, whereas openings are rare when glycine is absent so all desensitization must proceed through the slower closed-to-desensitized pathway.

We have made several main assumptions in our analysis, and we wish to stress them here. First, we have assumed that a state diagram with only four states (resting, closed, open, and desensitized) is adequate. Second, in our estimate of how many openings were missed, we have supposed that the open time distribution (in range of 0-25 msec) is a single exponential. Finally, the NMDA receptor channel has been treated as if its behavior is markovian. We consider each of these assumptions in turn.

The NMDA receptor doubtless requires more than four states for its accurate representation (see, e.g., the kinetic schemes presented by Clements and Westbrook, 1991; Clements et al., 1992; Sather et al., 1992). Because desensitization is slower than the transitions between many of the other states, combining states that communicate by more rapid transitions, as we have done here, should provide a useful approximation. That our equations adequately describe the phenomena we are studying encourages this belief. Nevertheless, the rate constants we extracted must be recognized as relating to compound states, and quite different values could hold for individual transitions in more accurate state diagrams.

To estimate how many openings were undetected, we fitted the open time histograms with a single exponential and calculated how many openings were missed from the deviations from this exponential at short times. Clearly, a distinct, additional (but very brief) open state could have been present, and we would not have detected it. Further, desensitization might occur extremely rapidly from this hypothetical additional open state, such that our observed difference between desensitization rates of "closed" and "open" channels could arise entirely from the difference in the occurrence of these hypothetical states. We know of no data with which we can exclude this possibility (nor any evidence in favor of it), but we prefer the simpler analysis used above.

The conclusion that desensitization is more rapid from the channel's open state is based on the observation that more desensitization accumulated during epochs in which openings were seen. We have no direct evidence, however, that the open-todesensitized pathway is used. Perhaps the channel is not markovian, such that a closed channel remembers previous openings in such a way that the channel increases its desensitization rate for a period after it has visited an open state. This possibility is very difficult to exclude without a much greater quantity of data at higher resolution than we have available. We note, how- 
ever, that the fact that the accumulation of desensitization is well described by the differential equations that suppose markovian channels supports the notion that the channels possess no strong history dependency.

Jahr (1992), using a quite different method from the one we have employed, determined the maximum open probability to be about 0.27 , a value in good agreement with our estimate of 0.29 for upper limit for this quantity. Thus, the maximum open probability for NMDA receptor channels, at least of the types investigated to date, is unlikely to be close to unity. If the Jahr estimate is accurate, our agreement with his value supports the validity of our estimates for the number of channels in the patches because our method makes use of this quantity, whereas his method does not.

We have been less successful than we would have liked in answering all of the open questions about desensitization; a fully successful treatment of this problem would include a detailed state diagram with rates for all of the possible transitions and would encompass all of the forms of desensitization. Nevertheless, the approach we have used here should be of utility in other contexts. Further, we have provided evidence that the pathways leading to the desensitized state are more complex than has previously been assumed. Finally, the actual desensitization process must be at least as complicated as what we have described, so kinetic models of NMDA receptor function should, at a minimum, contain the two pathways to desensitization.

\section{References}

Benveniste M, Vyklicky L Jr, Mayer ML, Clements J (1990) A kinetic analysis of the modulation of $N$-methyl-D-aspartic acid receptors by glycine in mouse cultured hippocampal neurons. J Physiol (Lond) 428:333-357.

Carbone E, Lux HD (1987) Kinetics and selectivity of a low-voltageactivated calcium current in chick and rat sensory neurons. J Physiol (Lond) 386:547-570.

Chizhmokov IV, Kiskin NI, Krishtal OA (1992) Two types of steadystate desensitization of $N$-methyl-D-asparate receptor in isolated hippocampal neurons of the rat. J Physiol (Lond) 448:453-472.

Clements JD, Westbrook GL (1991) Activation kinetics reveal the number of glutamate and glycine binding sites on the $N$-methyl-DAspartate receptor. Neuron 7:605-613.

Clements JD, Lester RAJ, Tong G, Jahr CE, Westbrook GL (1992) The time course of glutamate in the synaptic cleft. Science 258:14981501.

Colquhoun D, Sigworth FJ (1983) Fitting and statistical analysis of single-channel records. In: Single-channel recordings (Sakmann B, Neher E, eds), pp 191-263. New York: Plenum.
Gibb AJ, Colquhoun D (1991) Glutamate activation of a single NMDA receptor-channel produces a cluster of channel openings. Proc R Soc Lond [Biol] 243:39-45.

Jahr CE (1992) High probability opening of NMDA receptor channels by L-glutamate. Science $255: 470-472$.

Jahr CE, Stevens CF (1987) Glutamate activates multiple single-channel conductances in hippocampal neurons. Nature 325:522-525.

Konnerth A, Lux HD, Morad M (1987) Proton-induced transformation of calcium channel in chick dorsal root ganglion cells. J Physiol (Lond) 386:603-633.

Legendre P, Rosenmund C, Westbrook GL (1993) Inactivation of NMDA channels in cultured hippocampal neurons by intracellular calcium. J Neurosci 13:674-684.

Lester RA, Jahr CE (1992) NMDA channel behavior depends on agonist affinity. J Neurosci 12:635-643.

Lester RAJ, Clement JD, Westbrook GL, Jahr CE (1990) Channel kinetics determine the time course of NMDA receptor-mediated synaptic currents. Nature 346:565-567.

Lester RAJ, Tong G, Jahr CE (1993) Interactions between the glycine and glutamate binding sites of the NMDA receptor. J Neurosci 13: $1088-1096$.

Mayer ML, Westbrook GL (1985) The action of $N$-methyl-D-aspartic acid on mouse spinal neurones in culture. J Physiol (Lond) 361:6590.

Mayer ML, MacDermott AB, Westbrook GL, Smith SJ, Barker JL (1987) Agonist- and voltage-gated calcium entry in cultured mouse spinal cord neurones under voltage clamp measured using arsenazo III. J Neurosci 7:3230-3244.

McManus OB, Spivak CE, Blatz AL, Weiss DS, Magleby KL (1989) Fractal models, Markov model and channel kinetics. Biophys J 55: 383-385.

Press WH, Flannery BP, Teukolsky SA, Vetterling WT (1989) $\mathrm{Nu}$ merical recipes, The art of scientific computing (Fortran), pp 498528. Cambridge: Cambridge UP.

Sather W, Johnson JW, Henderson G, Ascher P (1990) Glycine-insensitive desensitization of NMDA responses in cultured mouse embryonic neurons. Neuron 4:725-731.

Sather W, Dicudonne S, MacDonald JF, Ascher P (1992) Activation and desensitization of $N$-methyl-D-asparate receptors in nucleated patches from mouse neurons. J Physiol (Lond) 450:643-672.

Shirasaki T, Nakagawa T, Wakamori M, Tateishi N, Fukuda A, Murase $\mathrm{K}$, Akaike N (1990) Glycine-insensitive desensitization of $N$-methyl-D-aspartate receptors in acutely isolated mammalian central neurones. Neurosci Lett 108:93-98.

Sigworth FJ (1980) The variance of sodium current fluctuations at the node of Ranvier. J Physiol (Lond) 307:97-120.

Zilberter Y, Uteshev V, Sokolova S, Khodorov B (1991) Accelerated communication: desensitization of $N$-methyl-D-aspartate receptors in neurons dissociated from adult rat hippocampus. Mol Pharmacol 40: 337-341. 